\title{
Revista Ambiental
}
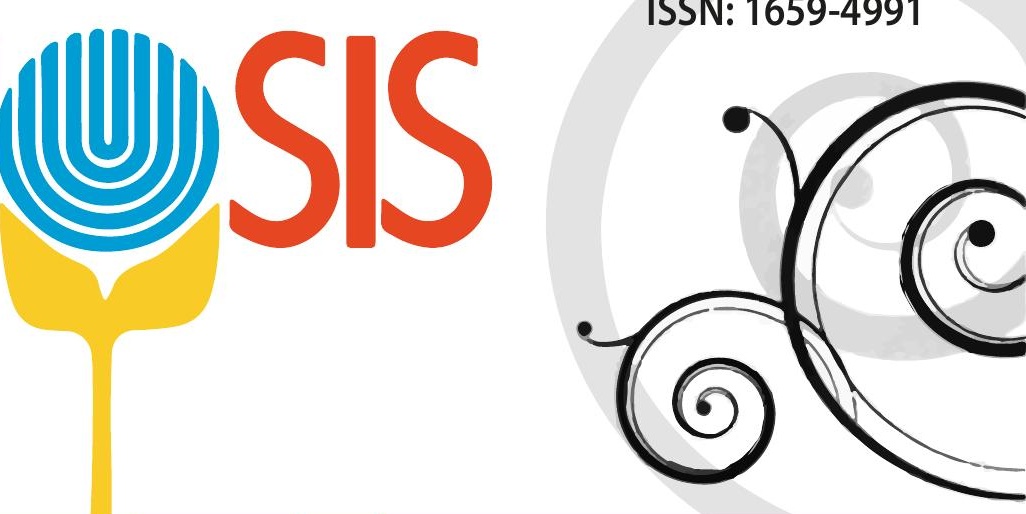

Volumen 32/ Número 1/ junio 2021

\section{Universidad Estatal a Distancia / Centro de Educación Ambiental}

\section{Equipo editorial}

\section{Directora}

Wendy Garita Azofeifa

Universidad Estatal a Distancia, Costa Rica.

\section{Editora}

Soledad Urbina Vargas

Universidad Estatal a Distancia, Costa Rica.

\section{Consejo Editorial}

Wendy Garita Azofeifa

Universidad Estatal a Distancia, Costa Rica.

Soledad Urbina Vargas

Universidad Estatal a Distancia, Costa Rica.

Sonia Rojas Vargas

Universidad Estatal a Distancia, Costa Rica.

Oscar Chacón Chavarría

Universidad Estatal a Distancia, Costa Rica.

Lucía Ortiz Montoya

Universidad Estatal a Distancia, Costa Rica.

\section{Consejo Asesor}

Jacqueline García Fallas

CIEA-UCR

Gladys Jiménez Valverde IMN

Patricia Rojas Núñez

CIEA-UCR

Federico Alice Guier

UNA

José Millán Araujo PRIGA-UNA

Henry Arias Guido

MEP

Bernal Herrera Fernández FUNDECOR-UICN

Andrea Suárez Serrano

UNA

Monserrat Espinach Rueda

UNED

Carmen Roldán Chacón

FONAFIFO

Marvin Torres Hernández UTN

Lorena Arias Zúñiga

ITCR

Carlos Calleja Amador ITCR

César Sancho Solís

UNED

\section{Adrián Ruiz Rodríguez} UNED

Fiorella Donato Calderón Jubilada-UNED

Olga Durán Monge

SINAC

Biocenosis es una publicación semestral del Centro de Educación Ambiental (CEA) de la Universidad Estatal a Distancia, editada desde 1979. El propósito de la revista es contribuir con el proceso de la sensibilización a la acción por medio de la educación ambiental y servir como medio de comunicación internacional entre la comunidad científica y los estudiantes universitarios, así como el público general. Es una revista de divulgación científica y cultural que provee acceso libre y abierto, bajo el principio de hacer disponible la investigación y fomentar el intercambio de conocimiento. Todos los trabajos publicados se pueden descargar de forma libre, y se pueden utilizar y reutilizar de acuerdo con la licencia Creative Commons Atribución-NoComercial 4.0 Internacional.

Contacto: Centro de Educación Ambiental, Universidad Estatal a Distancia. Sede Central, edificio A, piso 2. De la Rotonda Betania 500m al este, carretera a Sabanilla, Mercedes de Montes de Oca. Ap. postal 474-2050.

Teléfono +5062527 2255 • Correos: biocenosis@uned.ac.cr • surbina@uned.ac.cr

Fotografía de portada: Luis José Venegas. Nosara, Guanacaste, marzo 2019. 
Wendy Garita Azofeifa

1. Amenazas que enfrentan los monos congo (Alouata palliata) en Costa Rica

e iniciativas de conservación para el bienestar y una coexistencia sana con la especie

Threats faced by howler monkeys (Alouata palliata) in Costa Rica and conservation

initiatives for their well-being and our healthy coexistence with the species

Gabriela Jones Román

Cinthia Villalobos Suárez

Rose Marie Menacho Odio

2. Experiencia académica escolar: siembra y seguimiento de plantas nativas

para la recuperación ambiental del Macizo de Iguaque, Villa de Leyva, Colombia

School academic experience: planting and monitoring native plants for the environmental recovery

of the Iguaque Massif, Villa de Leyva, Colombia.

Ivonne Lorena Reina Fonseca

Andrés Felipe Cuadrado Hernández

Juan Carlos Salas Castañeda

Javier Ernesto Cortés-Suárez

3. La educación ambiental: una herramienta para promover el bienestar de las aves silvestres

Environmental Education: a tool to promote wild birds' welfare

Rose Marie Menacho-Odio

Sonia Rojas Vargas

4. Anfibios en peligro: amenazas y estrategias efectivas de conservación

Endangered amphibians: threats and effective conservation strategies

Juan Gabriel Abarca Alvarado

5. Los hongos como elementos clave en la productividad del suelo, la agricultura y el bienestar social

Fungi as key elements in soil productivity, agriculture, and social wellness.

Krystal Zúñiga-Castro

Gerald Quirós-Cedeño

6. Las tecnologías de la información y la comunicación como herramientas para la educación ambiental

Information and communication technologies as tools in environmental education

Óscar Chacón Chavarría

7. Educación ambiental desde el trabajo comunal universitario: experiencia de la Reserva Biológica Alberto Manuel Brenes, San Ramón, Costa Rica

Environmental education from university communal work: the experience of the Alberto Manuel Brenes Biological Reserve, San Ramón, Costa Rica.

Melvin Cartín Núñez

8. Levantamiento de información geoespacial con la aplicación Input para el manejo de residuos sólidos, Upala Costa Rica

Geospatial data collection with the Input Application for solid waste management, Upala, Costa Rica......

Luis Diego Mora-Jiménez

9. La educación ambiental: una estrategia necesaria para la conservación de la geodiversidad del área serrana de Tandil, Argentina

Environmental education: a necessary strategy for the conservation of geodiversity in the mountainous area of Tandil, Argentina

Aldo Ramos Schenck

10. Factores de éxito para la gestión de residuos sólidos valorizables en la Universidad Nacional de Costa Rica, Sede Central en Heredia Success factors for the management of recoverable solid residues at the Universidad Nacional de Costa Rica, Heredia, Costa Rica

Gerlin Salazar-Vargas

Rooel Campos-Rodríguez

Noelia Garita Sánchez 


\section{EDITORIAL}

Con gran entusiasmo, presentamos el volumen 32 (1) de la revista Biocenosis, el cual refleja la interdisciplinariedad que complementa el quehacer de la educación ambiental y su evolución a través de los años, disciplina clave y pilar para el mantenimiento del entorno y la convivencia sana con las especies con las que compartimos el planeta.

En la publicación, brindamos a los lectores el abordaje de varios temas actuales como las amenazas que enfrentan los monos congo, especie que se encuentra en peligro de extinción debido a factores como la pérdida de su hábitat, la fragmentación del bosque, la pérdida de conectividad y un ambiente modificado por infraestructuras antropogénicas. De igual manera, se muestra la preocupación por la realidad que viven los anfibios, como uno de los grupos más amenazados por una serie de factores, ambientales y humanos, que ha reducido la supervivencia de estas frágiles especies, así como un artículo que presenta algunas problemáticas ambientales que afectan a las aves silvestres y las recomendaciones para promover su buen manejo, bienestar y conservación.

El uso de las tecnologías de información y comunicación aplicadas como herramientas educativas en este nuevo contexto pandémico que vivimos, es otro de los artículos que compartimos con los lectores con el objetivo de dar a conocer el uso y la funcionalidad de una serie de herramientas tecnológicas que permiten el fortalecimiento y la adaptación de los procesos educativos.

La gestión integral de los residuos, otra área temática urgente de trabajar en la actualidad, se aborda mediante un caso sobre el análisis de los factores de éxito de una universidad pública en la gestión de residuos valorizables. También, por medio de una experiencia interesante y novedosa, gracias al levantamiento de información geoespacial con una aplicación, se promueve mejorar la planificación en la ampliación de la cobertura del servicio de recolección de residuos sólidos en un cantón del norte del país. Por último, en este volumen se incluyen también artículos que muestran diversas experiencias educativas como estrategias de recuperación ambiental, conservación de la geodiversidad y promoción de acciones ambientales en un área protegida.

Esperamos que los contenidos, presentados bajo el enfoque que mantiene la revista de informar y sensibilizar a nuestro público meta, cumplan con el objetivo de contribuir con el proceso de la sensibilización a la acción por medio de una educación ambiental fortalecida con las diversas áreas disciplinarias afines que complementan su quehacer.

Wendy Garita Azofeifa 